\title{
Development of a delirium risk screening tool for long-term care facilities
}

Jane McCusker, MD, DrPH, ${ }^{\mathrm{a}, \mathrm{b}}$ Martin G. Cole, MD, ${ }^{\mathrm{a}, \mathrm{c}, \mathrm{d}}$ Philippe Voyer, RN, PhD, ${ }^{\mathrm{e}}$ Antonio Ciampi, $\mathrm{PhD}$, ${ }^{\mathrm{a} b}$ Johanne Monette, MD, MSc, ${ }^{\mathrm{f}}$ Nathalie Champoux, MD, MSc, ${ }^{\mathrm{g}}$ Minh Vu, MD, ${ }^{\text {,i }}$ and Eric Belzile, $\mathrm{MSc}^{\mathrm{a}}$

${ }^{a}$ St. Mary's Research Centre, St. Mary’s Hospital, Montreal, Canada; Departments of ${ }^{b}$ Epidemiology, Biostatistics, and Occupational Health and ${ }^{\mathrm{d}}$ Psychiatry, McGill University, Montreal, Canada

${ }^{\mathrm{c}}$ Department of Psychiatry, St. Mary’s Hospital Center, Montreal, Canada

${ }^{\mathrm{e}}$ Faculty of Nursing Sciences, Laval University, Sainte-Foy, Canada

${ }^{\mathrm{f}}$ Division of Geriatric Medicine, Jewish General Hospital and Maimonides Geriatric Center

${ }_{\mathrm{g}}^{\mathrm{g}}$ Institut Universitaire de Gériatrie de Montréal, Department of Family Medicine, Université de Montréal, Montreal, Canada

${ }^{\mathrm{h}}$ Division of Geriatric Medicine, Centre Hospitalier de l’Université de Montréal, Montreal, Canada

${ }^{\mathrm{i} D}$ Department of Medicine, Université de Montréal, Montreal, Canada

Corresponding author: Dr. J. McCusker, St. Mary’s Research Centre, Hayes Pavilion, Suite 3734, 3830 Avenue Lacombe, Montreal, Quebec, H3T 1M5, Canada. E-mail: jane.mccusker@mcgill.ca

This work was funded by: the Canadian Institutes for Health Research, Institute of Aging (IAO 69519); the Alzheimer Society of Canada with the Canadian Nurses Foundation and the Nursing Care Partnership of the Canadian Health Services Research Foundation (ASC 0791); the Canadian Institutes for Health Research Institutes of Aging and of Gender and Health (CRG 82953); and the Canadian Institutes for Health Research, Institute of Aging with the Alzheimer Society of Canada and the Canadian Nurses Foundation (CPC 102348).

Objectives: To develop a delirium risk screening tool for use in longterm care (LTC) facilities.

Methods: The sample comprised residents aged 65 and over of 7 LTC facilities in Montreal and Quebec City, Canada, admitted for LTC. Primary analyses were conducted among residents without delirium at baseline. Incident delirium was diagnosed using multiple data sources during the 6-month follow-up. Risk factors, all measured at or prior to baseline, included the following 6 groups: sociodemographic, medical, cognitive status, physical function, agitated behaviour, and symptoms of depression. Variables were analyzed individually and by group using Cox regression models. Clinical judgement was used to select the most feasible among similarly performing factors.

Results: The cohort comprised 206 residents without delirium at baseline; 69 cases of incident delirium were observed (rate 7.6 per 100 person-weeks). The best-performing screening tool comprised 5 items, with an overall area under the curve of 0.82 (95\% CI 0.76, 0.88). These items included brief measures of cognitive status, physical function, behavioral, and emotional problems. Using cut-points of 2 (or 3) over 5, 
the scale has a sensitivity of $90 \%$ (63\%), specificity of 59\% (85\%), and positive predictive value of 52\% (66\%).

Conclusion: This brief screening tool allows nurses to identify LTC residents at increased risk for delirium. These residents can be targeted for closer monitoring and preventive interventions.

Key words: delirium, risk factor, screening, long-term care

\section{INTRODUCTION}

Delirium is a frequent and serious problem in long-term care (LTC) and post-acute care facilities, ranging in prevalence from 3.4\% to 70.3\% (Fries, et al. 1993; Kiely, et al. 2003; Marcantonio, et al. 2003; McCusker, et al. 2011a, b; Sandberg, et al. 1998; Voyer, et al. 2009). In post-acute settings, delirium is a risk factor for mortality (Kiely, et al. 2007; Kiely, et al. 2009) and in LTC it is a risk factor for adverse outcomes, particularly cognitive decline (McCusker et al. 2011b). Thus, efforts are warranted to prevent delirium in LTC settings. A brief screening tool that could be used by LTC nurses to identify residents at increased risk of delirium would facilitate the targeting of delirium interventions to highrisk residents (Inouye, et al. 1999; Inouye, et al. 2000).

Risk screening tools have been developed previously for delirium in acutecare medical and surgical settings,(Inouye, et al. 1993; Marcantonio, et al. 1994; Rudolph, et al. 2009) and for other important geriatric problems, such as functional decline and institutionalization (McCusker, et al. 1999; McCusker, et al. 2002). Because risk factors for delirium may differ from those in acute care, (McCusker et al. 2011a) the development of a delirium risk screening tool for LTC should be based on a careful examination of risk factors in LTC populations. Feasibility should also be considered; ideally, the screening tool should be short and comprised of risk factors that are either already known to LTC nurses or can be abstracted from the medical record or quickly assessed.

The delirium risk screening tool was designed be used by LTC nurses in conjunction with a delirium screening tool using one of two strategies: 1 ) residents are screened first for delirium; only those without delirium receive the risk screening tool; or 2) residents are screened first with the risk screening tool; those at increased risk would then be screened for delirium. In either strategy, high-risk residents without prevalent delirium would be assessed for potentially modifiable risk factors for delirium (e.g., medications, and environmental factors such as aids to orientation, and use of restraints) and would be monitored periodically for delirium.

The primary objective of this study was to develop a brief risk screening tool to predict incident delirium among LTC residents aged 65 and over without prevalent delirium (strategy 1 above). The second objective was to describe the concurrent validity of the screening tool to detect prevalent delirium (strategy 2 above) (Dendukuri, et al. 2004). 


\section{METHODS}

We developed the screening tool in collaboration with two committees: an advisory committee comprising clinicians and administrators of LTC facilities in two Canadian provinces; and a committee of LTC nurse clinicians in Quebec City. The data source, welldescribed previously, (McCusker et al. 2011a, b) was a prospective, observational, cohort study of LTC residents at 7 study sites in Montreal and Quebec City. The sites varied in size (111 - 415 LTC beds), funding (private or government), daily hours of care/resident (3 or more hours), and background of nursing staff [33-100\% were registered nurses (RNs)]. At some sites, auxiliary nurses with less training than RNs assist with various nursing tasks (e.g., distribution of medicines, blood samples, skin care, and measurement of vital signs). In addition to nursing staff, nurses' aides assist residents with activities of daily living and emotional support.

We recruited residents who were aged 65 or over, and admitted for LTC. We used stratified sampling with two strata: 1) at most moderate cognitive impairment [Mini-Mental State Exam (MMSE) score 10 or more], and 2) severe cognitive impairment (MMSE score less than 10). Both newly admitted and longer term residents were recruited consecutively from resident lists. Residents were excluded only if: 1) they were unable to communicate in English or French; or 2) the primary nurse or research assistant (RA) did not have time to complete assessments because of a high workload. Competence to consent to the study was based on the clinical impression of the primary nurse. Competent residents were invited to participate in the study by a trained research assistant (RA). Among incompetent residents, a letter describing the study was sent to the legal guardian if available, or (because many LTC residents are not legally declared incompetent), to the responsible family member. The legal guardian/family member informed the nurse if they were willing to meet the RA. The study protocol was approved by the research ethics boards of McGill University and those sites with a review committee.

Residents were followed with repeated weekly assessments by the RA for up to 24 weeks or until death or transfer to another facility. The primary nurse (who was blind to the RA assessments) was interviewed at baseline and monthly thereafter. The initial RA and nurse assessments included measures of delirium symptoms and of risk factors; the followup assessments included measures of delirium symptoms (see below). At the end of the study, data on medical history before study enrolment and on potential symptoms of delirium during the follow-up period were abstracted from resident charts by research clinicians, blind to the RA assessments.

The data source for this study, well described previously, ${ }^{1,7}$ was a prospective, observational, cohort study of LTC residents at seven study sites in Montreal and Quebec City. Residents aged 65 and older admitted for LTC were recruited. Stratified sampling with two strata were used: no to moderate cognitive impairment (Mini-Mental State Examination (MMSE) score $\geq 10$ ) and severe cognitive impairment (MMSE score $<10$ ). Newly admitted and longer-term residents were recruited consecutively from resident lists. Residents were excluded only if they were unable to communicate in English or French or 
2) the primary nurse or research assistant (RA) did not have time to complete assessments because of a high workload. Competence to consent to the study was based on the clinical impression of the primary nurse. Competent residents were invited to participate in the study by a trained research assistant (RA). Among incompetent residents, a letter describing the study was sent to the legal guardian if available, or (because many LTC residents are not legally declared incompetent), to the responsible family member. The legal guardian/family member informed the nurse if they were willing to meet the RA. The study protocol was approved by the research ethics boards of McGill University and those sites with a review committee.

Residents were followed with repeated weekly assessments by the RA for up to 24 weeks or until death or transfer to another facility. The primary nurse (who was blind to the RA assessments) was interviewed at baseline and monthly thereafter. The initial RA and nurse assessments included measures of delirium symptoms and of risk factors; the followup assessments included measures of delirium symptoms (see below). At the end of the study, data on medical history before study enrolment and on potential symptoms of delirium during the follow-up period were abstracted from resident charts by research clinicians, blind to the RA assessments.

\section{Measurement of delirium}

Our primary measure of delirium, based on Confusion Assessment Method (CAM) criteria for probable delirium, required the presence of: 1) either acute onset or fluctuation, 2) inattention, and 3) either disorganized thinking or altered consciousness (Inouye, et al. 1990). These symptoms were detected either by RAs or nurses, and the CAM algorithm was applied (Inouye et al. 1990). In comparison with the use of symptoms observed by an RA only, this measure results in a higher estimate of the prevalence of delirium and has somewhat better overall predictive validity for adverse outcomes at 6 months (McCusker et al. 2011b).

\section{Measures of risk factors}

Measures of potential risk factors were derived from the RA baseline assessment, the primary nurse baseline interview, and resident charts covering the period between admission and the baseline interview. We restricted the risk factors to those with clinical face validity that could easily be assessed by nurses, single items rather than multi-item scale scores, and resident characteristics that were not easily modifiable.

A total of 136 potential risk factors (available upon request) were considered in the following 6 groups (numbers of items per group shown in parentheses): sociodemographic (5), medical problems (19), cognition (42), physical function (21), behaviour (29), and mood (20). Sociodemographic items included age, sex, country of birth, months since admission, and location prior to admission. Medical problems included diagnoses recorded in the medical chart (Bravo, et al. 2002; Buntinx, et al. 2002). Cognition items included a chart diagnosis of dementia, and all items from the Mini-Mental Status Exam (Tombaugh 
and McIntyre 1992) and the Hierarchic Dementia Scale (Tombaugh and McIntyre 1992). Physical function items were derived from the Barthel Index,(Mahoney and Barthel 1965) days in bed in previous week, pain level, visual and hearing impairment, and falls. Behaviour items included those from the Nursing Home Behavior Problem Scale,(Ray, et al. 1992) and observations of agitation in the nursing notes. Mood items were derived from the Cornell Scale for Depression in Dementia, (Alexopoulos, et al. 1988a, b) and from observations of emotional disturbance in the nursing notes (e.g., cries, sad, irritable, impatient).

\section{Statistical methods}

A total of 280 residents were enrolled in the study. For the primary objective, 57 residents with prevalent delirium and 17 without at least one monthly follow-up were excluded, for a sample size of 206. For the second objective, 37 residents with an initial MMSE score less than 10 were removed.

Primary objective In preliminary analyses within each block of risk factors, we computed incidence rates (IR) per person/month of follow-up, incidence rate ratios (IRR), and hazard ratios (HR). We converted ordinal and categorical variables to binary variables, using the best cut-point with a minimum of 5 cases of incident delirium per risk category.

We selected variables for tool development using different approaches depending on the presence of multi-collinearity [defined as a variance inflation factor (VIF) of greater than 3]. When there was no multi-collinearity, we explored the data using univariate and multivariate Cox models (Allison 1995) for time to first delirium episode, over the study time (6 months). We selected variables with at least 5 cases of delirium detected in each risk group, plus one of the following criteria: $\mathrm{HR}>1.5$; or variables remaining in a reduced multivariate model with the lowest AIC (Akaike information criterion) and BIC ( Bayesian information criterion). When multi-collinearity existed, we performed Principal Components Analysis (PCA) with varimax rotation (Khattree and Naik 2000). We examined the relationship between each factor score and the outcome, and retained factors that, in univariate analyses, were statistically significant predictors of delirium. From each retained factor, we selected those variables with a factor loading $\geq 0.5$. In the case that several variables within a block met the above criteria (particularly a problem when considering multiple items from the same scale), we considered clinical coherence and feasibility to select those variables to be retained for the global model. For all Cox models, we tested constancy in time of the HR (Allison 1995) in order to verify the assumption of proportional hazards.

We based our model selection procedure on the Bayesian Model Averaging (BMA) approach, (Ibrahim, et al. 2001) which allows us to attach an a posteriori probability to model parameters. Our procedure, applied to the variables pre-selected as described above, consisted of four steps. First, a probability was calculated for each model, and the subset of models was retained using Occam's window algorithm with C=20 (Ibrahim et al. 2001; Madigan and Raftery 1994). (Essentially, if two models have the same explicative power, 
the algorithm selects the simplest of the two, i.e. the one with fewer variables). Second, for each given model containing exactly $k$ variables $(k=1,2, \ldots, 21)$, we examined the proportion of the retained models which contain those specific $\mathrm{k}$ variables, and selected the $\mathrm{k}$ with the highest proportion. Third, we considered all models with k variables and computed, for each variable of the original list, the average probability of having a non-zero coefficient. Finally, we used clinical judgment to select k variables among those with the highest average probability of being non-zero.

We created a candidate screening tool for a given group of k risk factors: the score of this risk tool is simply the sum of risk factors observed in a resident. Each screening tool was evaluated by calculating sensitivity, specificity, predictive value, receiver operating characteristic (ROC) curves, and area under the ROC curve (AUC) for predicting time of first delirium episode (Heagerty, et al. 2000).

Objective 2. The second objective, concerning the ability of the screening tool to identify prevalent delirium, was addressed in the original sample of 280, 57 of whom had delirium. Using the screening tool developed above, we computed the sensitivity, specificity, predictive value and AUC for predicting prevalent delirium (Beam 2002; Hanley and McNeil 1982).

Sensitivity analyses. In the primary sample without prevalent delirium (n=206): 1) we compared the performance of the tool in each participating facility; 2) we investigated the performance of the tool in the stratum with baseline MMSE scores of 10 or more; 3) because the optimal tool contained one paper and pencil task, we investigated the performance of a tool without this item.

Analyses were performed with SAS version 9.2 (PROC PHREG), STATA version 10.0 (ROCTAB) and R version 2.11.1, using age BMA(bic.surv) and survivalROC. 0

\section{RESULTS}

\section{Primary Objective: Development of Tool to Predict Incident Delirium}

Table 2 shows the 20 risk factors retained for tool development. In general, the IRR and HR values were similar. Most of the risk factors and those associated with the highest HRs (3 or more) were from the cognition group.

Table 3 shows the risk factors ranked in order of importance among all potential screening tools, based on the BMA procedure. Note that 3 risk factors (design copying, naming month, and emotional problems) were ranked the highest, with probabilities of $100 \%$ for inclusion in the best 5-item tools.

The statistically best performing tool comprised 5 items. Two of the items were from the MMSE (cannot name month, cannot copy design) and one each from nursing notes (emotional problems), the Barthel Index (completely dependent in personal hygiene), and the Cornell Scale (fidgets/restless). The overall AUC was 0.82 (95\% CI 0.76, 0.88) (Figure 1). Using cut-points of 2 (or 3 ) over 5 the scale has a sensitivity of $90 \%$ (63\%), specificity of $59 \%$ (85\%), and positive predictive value of 52\% (66\%). [Data available from the authors, upon request.] 


\section{Objective 2: Detection of Prevalent Delirium}

This analysis was conducted in the entire cohort of 280 residents, of whom 57 had prevalent delirium. The overall AUC was 0.73 (95\% CI 0.66, 0.80). At a cut-point of 2 or more, the sensitivity and specificity were $95 \%$ and $41 \%$, respectively. At a cut-point of 3 or more, these values were 58 and $67 \%$, respectively. The predictive value was $29 \%$ and $31 \%$ at cut-points of 2 and 3, respectively.

\section{Sensitivity Analyses}

Figure 2 shows the overall AUC (and 95\% CI) and the AUC in each of the 7 facilities participating in the study. Although there was variability among the facilities, only one (F) differed significantly from the others. This was a smaller (111 beds), private facility (all the others were publicly funded) with the highest proportion of RN-prepared nurses (100\%). Facility B was a large (320 beds) publicly funded nursing home with the lowest proportion of RN-prepared nurses (29\%) in the sample, and characterized by high turnover of nursing staff.

When residents with an initial MMSE score less than 10 at baseline $(n=37)$ were removed from the tool development cohort of $206(\mathrm{n}=169)$, the AUC with this sub-sample was similar to that in the development cohort of 206: 0.79 (95\% CI 0.72, 0.88).

Finally, to explore the performance of a more clinically feasible tool, we replaced the paper and pencil task (design copying) with the next best-performing item from Table 3 (talks, mutters, or mumbles to self). The AUC in the main sample of 206 of the alternative tool was 0.81 (95\% CI 0.75-0.87).

\section{DISCUSSION}

The primary objective of this study was to develop a brief, clinically feasible delirium risk screening tool with good performance characteristics, to help nursing staff to identify LTC residents at increased risk of incident delirium, among residents free of prevalent delirium. The best-performing screening tool comprised 5 items, with an overall AUC of 0.82 (95\% CI 0.76, 0.88). The tool comprises items that are either already known to nursing staff (dependence in personal hygiene, emotional problems and behavioural problems), or that can be quickly abstracted from the medical record or assessed at the bedside (ability to name the correct month or copy a design). Thus, the tool appears clinically feasible as well as possessing performance characteristics that compare favourably with those of delirium risk screening tools developed for acute care settings (AUC values between 0.71 and 0.81 ) (Inouye et al. 1993; Marcantonio et al. 1994; Rudolph et al. 2009). It should be noted, however, that the performance of screening tools may be higher in patient cohorts used for tool development than in those used for validation (Inouye et al. 1993; Marcantonio et al. 1994).

A secondary objective was to describe the ability of the screening tool to detect residents with prevalent delirium. This aspect of the screening tool would be important in facilities where nurses do not routinely used standardized tools to detect delirium. The 
overall AUC for this secondary outcome was 0.73 (95\% CI 0.66, 0.80), also an acceptable level of performance.

The performance of the tool in the prediction of incident delirium remained good across the 7 institutions participating in the study. These institutions varied in size, source of funding, and nursing staff. Notably, facility B with the lowest overall performance (AUC $=0.60$ ) was characterized by a low proportion of RNs and high staff turnover. Facility F with the highest overall performance (AUC $=0.99$ ) was characterized by a high proportion of RNs. The training of nurses and their familiarity with residents may affect the accuracy with which they reported delirium symptoms and risk factors in interviews, and in the charts. These findings suggest that the tool should be applied more cautiously in facilities with less highly nursing trained staff and high staff turnover. The sensitivity analysis in which residents with severe cognitive impairment (MMSE score less than 10) were removed from the sample also gave very similar results to those in the complete development cohort.

Those facilities who desire an even more clinically feasible tool (avoiding use of paper-and-pencil tests) may consider the option that we tested without this item. The performance of this tool was almost identical to that of the optimal tool. It should be noted however, that paper-and-pencil testing could be minimized when using the original tool, as nurses can first score those items that do not require bedside testing. The score cut-point (e.g. 2 or more) may be reached for some residents without the need for further testing.

This study demonstrates that predisposing risk factors for delirium in LTC are different from those in acute care. A 4-item delirium screening tool developed for acute care medical patients aged 70 and over included 4 risk factors: visual impairment, severe physical illness, cognitive impairment, high serum urea/creatinine ratio (Inouye et al. 1999). A 7-item screening tool to predict post-operative delirium among patients aged over 50 undergoing elective non-cardiac surgery included age 70 or over, cognitive impairment, functional impairment, alcohol abuse, and abnormal laboratory tests (Marcantonio et al. 1994). A 4-item screening tool to predict post-operative delirium among patients aged 60 and over undergoing cardiac surgery included: prior stroke or transient ischemic attack, cognitive impairment, serum albumin, and depression (Rudolph et al. 2009). While there are some risk factors common to acute and long-term care settings (cognitive and functional impairment and depression), measures of the clinical and physiological severity of illness appear to be more important in acute care settings. Reasons for this may be the absence of acute severe physical illness or lack of frequent laboratory testing in LTC settings. In contrast, measures of the severity of cognitive impairment and behavioral disturbance were more important in our LTC sample. This may be due to the higher prevalence of cognitive impairment in LTC, requiring measures of risk factors that distinguish residents by severity of cognitive impairment or the associated behavioral or emotional problems. It is notable that one of the strongest risk factors for delirium in this study was the MMSE designcopying test (2 overlapping pentagons). Abnormal performance in clock-drawing scores (an 
indicator of right hemisphere dysfunction) was a risk factor for delirium in 2 previous studies (Fisher and Flowerdew 1995; Mach, et al. 1996).

There are two main limitations of this study. First, because this was a secondary analysis, the measures of risk factors were those designed for the original study. They may need to be further adapted for use in LTC. Second, the sample size was sufficient for tool development but not for tool validation.

The following scenario describes the use of the tool in a facility that wishes to identify prevalent delirium and then implement monitoring and prevention protocols to prevent incident delirium. [This scenario is given for illustrative purposes only and will need to be confirmed in subsequent research.] The numbers in parentheses are estimates from the current study using a cut-point of 2 on the screening tool. The residents $(n=100)$ are first screened by nursing staff using the 5-item tool, either at admission, or whenever the screening program begins. We estimate that it would take only a few minutes to score the tool as much of the information will be known already to nursing staff. Sixty-six residents will screen positive. (This number is expected to vary by facility, and will depend primarily on the severity of cognitive impairment in the resident population.) These 66 residents could then be assessed for prevalent delirium (e.g., using the CAM); 19 would meet CAM criteria for delirium, and require intervention. The remaining 47 residents would be at high risk of incident delirium and require further assessment for potentially modifiable risk factors (e.g., medications, restraints) and preventive interventions. In a facility with fewer resources to detect residents at high risk and intervene, using a cut-point of 3 or more: 38 residents would be identified as high-risk, 12 would meet CAM criteria for delirium, and the remaining 26 residents would be at high risk of incident delirium.

\section{CONCLUSIONS}

The brief screening tool we developed may be used by nurses to identify LTC residents at increased risk of developing delirium. Identified residents may then be targeted for closer monitoring or preventive interventions. Validation studies should be conducted in other LTC settings.

Further research is needed on potentially modifiable (or precipitating) risk factors for delirium and the effectiveness of preventive interventions in LTC, as most prior research has been conducted in acute care. Research is also needed on the feasibility of implementing systematic risk screening for delirium in LTC.

The authors report no conflict of interest. The study funding agencies had no role in the collection, analysis, or interpretation of data, or in the writing of the report and the decision to submit for publication. 
TABLE 1 Characteristics of residents

\begin{tabular}{|c|c|c|}
\hline Variable & $\begin{array}{c}\text { Full } \\
\text { cohort }\end{array}$ & $\begin{array}{c}\text { Cohort for } \\
\text { development of tool }\end{array}$ \\
\hline $\mathrm{N}$ & 280 & 206 \\
\hline Mean Age & 85.4 & 85.0 \\
\hline Female (\%) & 56.4 & 58.7 \\
\hline Dementia (\%) & 65.6 & 60.2 \\
\hline Charlson comorbidity index score $4+(\%)$ & 30.0 & 28.6 \\
\hline MMSE Score (\%) & & \\
\hline$<10$ & 26.8 & 18.0 \\
\hline $10-17$ & 22.1 & 20.4 \\
\hline $17-24$ & 27.5 & 32.5 \\
\hline $24+$ & 23.6 & 29.1 \\
\hline Activities of Daily Living (BI) Score (\%) & & \\
\hline $0-19$ & 33.9 & 27.2 \\
\hline $20-59$ & 30.7 & 31.6 \\
\hline $60-100$ & 30.7 & 35.9 \\
\hline Missing & 4.6 & 5.3 \\
\hline CSDD Depression Scale Score 6+ (\%) & 19.6 & 13.1 \\
\hline 1+ Psychotropic meds (\%) & 55.0 & 51.0 \\
\hline Time in LTC (more than 1 year) & 66.4 & 63.1 \\
\hline
\end{tabular}

BI: Barthel Index CSDD: Cornell Scale for Depression in Dementia LTC: long-term care MMSE: Mini-Mental State Examination 
TABLE 2 Summary of Risk Factors Selected in Each Block $(\mathrm{n}=206)$

\begin{tabular}{|c|c|c|c|c|c|c|c|c|}
\hline Risk factor & & Source & Total (N) & $\begin{array}{c}\text { Incident } \\
\text { Delirium (N) } \\
\end{array}$ & $\mathbf{I R}^{*}$ & IRR** & H.R. & CI 95\% \\
\hline \multirow[t]{2}{*}{ Age } & $65-84$ & $\mathrm{CH}$ & 91 & 24 & 5.81 & 1.00 & 1.00 & \\
\hline & $85+$ & & 115 & 45 & 9.04 & 1.55 & 1.52 & $0.93-2.50$ \\
\hline Arrhythmia/pacemaker*** & Yes & & 57 & 22 & 9.21 & 1.32 & 1.29 & $0.78-2.15$ \\
\hline \multirow[t]{2}{*}{ Constipation } & No & $\mathrm{CH}$ & 180 & 56 & 7.08 & 1.00 & 1.00 & \\
\hline & Yes & & 26 & 13 & 10.83 & 1.53 & 1.55 & $0.85-2.83$ \\
\hline \multirow[t]{2}{*}{ Name month of the year } & Yes & RA (MMSE) & 95 & 13 & 2.71 & 1.00 & 1.00 & \\
\hline & No & & 111 & 56 & 12.99 & 4.80 & 4.41 & $2.41-8.08$ \\
\hline \multirow[t]{2}{*}{ Names a wrist watch } & Yes & RA (MMSE) & 173 & 47 & 5.89 & 1.00 & 1.00 & \\
\hline & No & & 33 & 22 & 19.47 & 3.31 & 2.97 & $1.78-4.94$ \\
\hline \multirow[t]{2}{*}{ Copies design } & Yes & RA (MMSE) & 72 & 7 & 1.80 & 1.00 & 1.00 & \\
\hline & No & & 134 & 62 & 11.85 & 6.57 & 6.00 & $2.74-13.12$ \\
\hline \multirow[t]{2}{*}{ Registers objects } & Yes & RA (HDS) & 179 & 51 & 6.16 & 1.00 & 1.00 & \\
\hline & No & & 27 & 18 & 21.69 & 3.52 & 3.05 & $1.77-5.24$ \\
\hline
\end{tabular}




\begin{tabular}{|c|c|c|c|c|c|c|c|c|}
\hline Risk factor & & Source & Total (N) & $\begin{array}{c}\text { Incident } \\
\text { Delirium (N) } \\
\end{array}$ & $\mathbf{I R}^{*}$ & $\begin{array}{c}\text { IRR }^{*} \\
*\end{array}$ & H.R. & CI $95 \%$ \\
\hline \multirow[t]{2}{*}{ Can draw } & Yes & RA (HDS) & 175 & 53 & 6.65 & 1.00 & 1.00 & \\
\hline & No & & 31 & 16 & 14.04 & 2.11 & 1.94 & $1.11-3.40$ \\
\hline Independent feeding & No & & 130 & 55 & 10.44 & 2.86 & 2.65 & $1.47-4.77$ \\
\hline \multirow[t]{2}{*}{$\begin{array}{l}\text { Independent / partial assistance for } \\
\text { personal hygiene }\end{array}$} & Yes & NI (BI) & 126 & 28 & 4.58 & 1.00 & 1.00 & \\
\hline & No & & 80 & 41 & 13.71 & 3.00 & 2.76 & $1.70-4.50$ \\
\hline Falls & Yes & & 10 & 5 & 12.50 & 1.70 & 1.67 & $0.67-4.15$ \\
\hline \multicolumn{9}{|l|}{ Block 5: Behavioural problems } \\
\hline \multirow[t]{2}{*}{ Resists care } & No & NI (NHBPS) & 166 & 47 & 6.20 & 1.00 & 1.00 & \\
\hline & Yes & & 40 & 22 & 14.30 & 2.31 & 2.17 & $1.31-3.61$ \\
\hline \multirow[t]{2}{*}{ Fidgets, unable to sit still, restless } & No & NI (NHBPS) & 185 & 55 & 6.55 & 1.00 & 1.00 & \\
\hline & Yes & & 21 & 14 & 19.72 & 3.01 & 2.76 & $1.53-4.97$ \\
\hline \multirow[t]{2}{*}{ Talk, mutters, or mumbles to herself } & No & NI (NHBPS) & 167 & 44 & 5.71 & 1.00 & 1.00 & \\
\hline & Yes & & 39 & 25 & 17.73 & 3.11 & 2.85 & $1.74-4.66$ \\
\hline
\end{tabular}




\begin{tabular}{|c|c|c|c|c|c|c|c|c|}
\hline \multirow[b]{2}{*}{ Risk factor } & & \multirow[b]{2}{*}{ Source } & \multirow[b]{2}{*}{ Total (N) } & \multirow{2}{*}{$\begin{array}{c}\text { Incident } \\
\text { Delirium (N) }\end{array}$} & \multirow[b]{2}{*}{ IR* } & \multirow{2}{*}{$\begin{array}{c}\text { IRR* } \\
*\end{array}$} & \multicolumn{2}{|c|}{ Univariate model } \\
\hline & & & & & & & H.R. & CI $95 \%$ \\
\hline \multirow[t]{2}{*}{ Agitation } & No & NN & 183 & 57 & 6.95 & 1.00 & 1.00 & \\
\hline & Yes & & 23 & 12 & 13.19 & 1.90 & 1.82 & $0.98-3.40$ \\
\hline \multicolumn{9}{|l|}{ Block 6: Depression } \\
\hline \multirow[t]{2}{*}{ Irritability } & No & NI (CSDD) & 150 & 44 & 6.42 & 1.00 & 1.00 & \\
\hline & Yes & & 56 & 25 & 11.06 & 1.72 & 1.66 & $1.02-2.72$ \\
\hline \multirow[t]{2}{*}{ Agitation } & No & NI (CSDD) & 173 & 50 & 6.35 & 1.00 & 1.00 & \\
\hline & Yes & & 33 & 19 & 15.32 & 2.41 & 2.29 & $1.35-3.88$ \\
\hline \multirow[t]{2}{*}{ Loss of interest } & No & NI (CSDD) & 188 & 60 & 7.13 & 1.00 & 1.00 & \\
\hline & Yes & & 18 & 9 & 13.04 & 1.83 & 1.73 & $0.86-3.49$ \\
\hline \multirow[t]{2}{*}{ Emotional problems } & No & $\mathrm{NN}$ & 191 & 60 & 6.98 & 1.00 & 1.00 & \\
\hline & Yes & & 15 & 9 & 17.31 & 2.48 & 2.28 & $1.13-4.59$ \\
\hline
\end{tabular}

*IR: Incidence rate per 100 percent weeks

**IRR: Incidence rate ratio

*** Multivariate model in block \#2 HR: 1.76 [0.99 ; 3.15]

BI: Barthel Index CH: Chart review CI: Confidence interval CSDD: Cornell Scale for Depression in Dementia HDS: Hierarchic Dementia Scale HR: Hazard ratio MMSE: Mini-Mental State Examination

NI: Nursing Interview form (baseline) NHBPS: Nursing Home Behavioral Problem Scale NN: Nursing Notes

RA: Resident assessment form (baseline) 
TABLE 3 Ranking of Best Risk Factors Among Models Containing 5 Variables

\begin{tabular}{|c|c|c|c|}
\hline Ranking & Block \# & Risk factor & $\begin{array}{c}\text { BMA }^{*} \\
\mathrm{n}=128 \text { models }\end{array}$ \\
\hline 1 & 3 & Cannot copy design & $100 \%$ \\
\hline 1 & 3 & Cannot name month & $100 \%$ \\
\hline 1 & 6 & Emotional problems & $100 \%$ \\
\hline 2 & 5 & Talks, mutters, or mumbles to herself & $44 \%$ \\
\hline 3 & 4 & Completely dependent in personal hygiene & $39 \%$ \\
\hline 4 & 5 & Fidgets, is unable to sit still, restless & $23 \%$ \\
\hline 5 & 6 & Agitation & $16 \%$ \\
\hline 6 & 6 & Irritability & $12 \%$ \\
\hline 7 & 5 & Resists care & $10 \%$ \\
\hline 8 & 4 & No participation in activities & $9 \%$ \\
\hline 9 & 4 & Requires feeding assistance & $7 \%$ \\
\hline 10 & 2 & Arrhythmia or pacemaker & $6 \%$ \\
\hline 10 & 3 & Cannot name wrist watch & $6 \%$ \\
\hline 11 & 3 & Cannot register objects & $5 \%$ \\
\hline 12 & 8 & Falls & $4 \%$ \\
\hline 13 & 5 & Agitation & $3 \%$ \\
\hline 13 & 2 & Constipation & $3 \%$ \\
\hline 13 & 3 & Cannot draw & $3 \%$ \\
\hline 13 & 6 & Loss of interest & $3 \%$ \\
\hline 13 & 1 & Age $85+$ & $3 \%$ \\
\hline 13 & 3 & Dementia & $3 \%$ \\
\hline
\end{tabular}


FIGURE 1 Receiver Operating Characteristic (ROC) Curve for the Prediction of Incident Delirium $(n=206)$

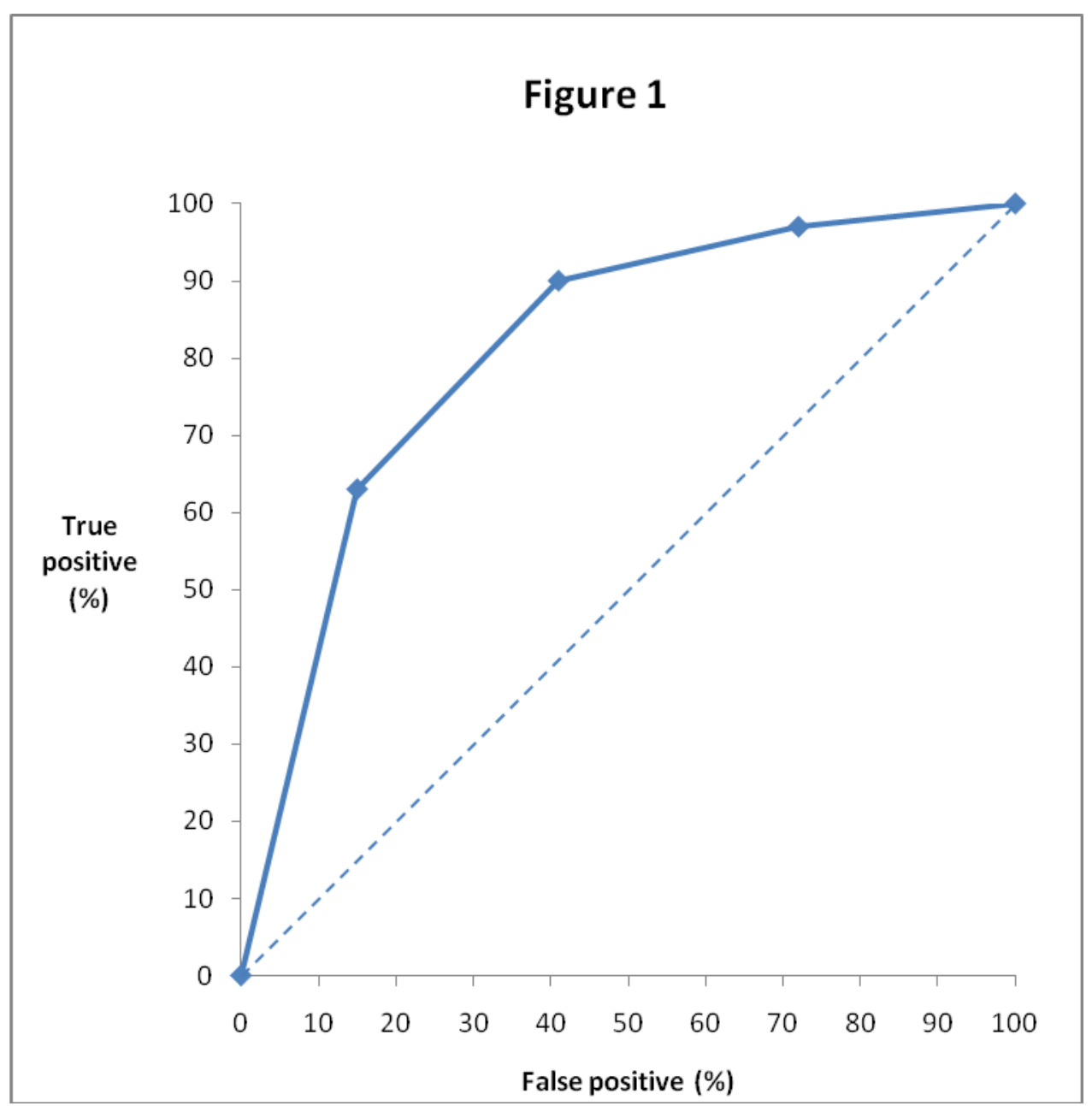

Area under the curve (95\% confidence interval): $0.82(0.76 ; 0.88)$ 
FIGURE 2 Performance of the Screening Tool by Institution (Area under the curve and 95\% confidence intervals)

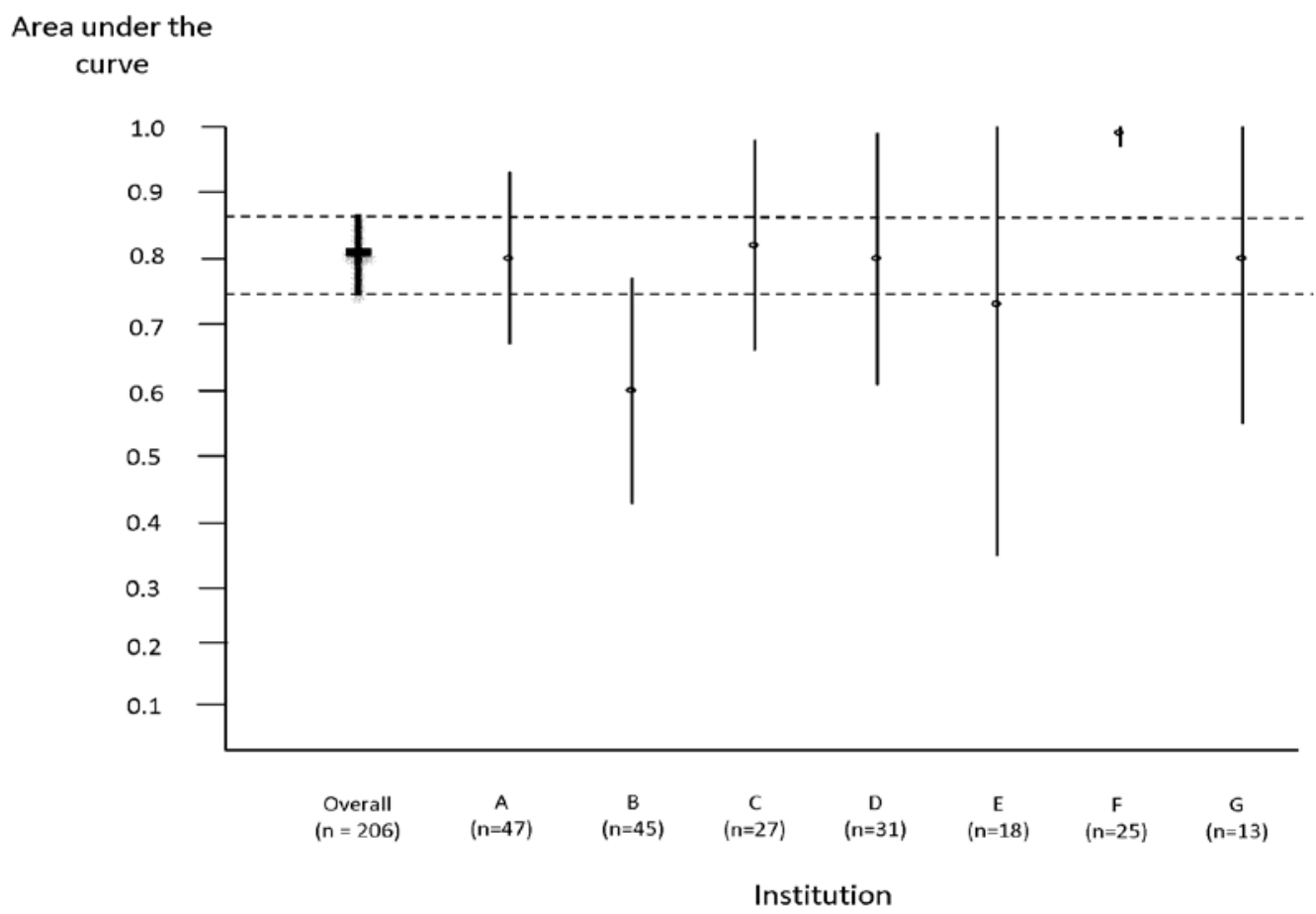

Dotted lines represent the lower and upper bounds of the 95\% confidence interval of the overall AUC 


\section{REFERENCES}

Alexopoulos GS, Abrams RC, Young RC, et al. 1988a. Cornell scale for depression in dementia. Biol Psychiatry 23: 271-284.

Alexopoulos GS, Abrams RC, Young RC, et al. 1988b. Use of the Cornell Scale in nondemented patients. J Am Geriatr Soc 36: 230-236.

Allison PD. 1995. Survival analysis using SAS. A practical guide. Cary, North Carolina: SAS Institute Inc.

Beam C. 2002. Biostatistical applications in cancer research. Springer, Netherlands: Kluwer Academic Publisher.

Bravo G, Dubois M-F, Hébert R, et al. 2002. A prospective evaluation of the Charlson Comorbidity Index for use in long-term care patients. J Am Geriatr Soc 50: 740-745.

Buntinx F, Niclaes L, Suetens C, et al. 2002. Evaluation of Charlson's comorbidity index in elderly living in nursing homes. J Clin Epidemiol 55: 1144-1147.

Dendukuri N, McCusker J,Belzile E. 2004. The Identification of Seniors At Risk screening tool: Further evidence of concurrent and predictive validity. J Am Geriatr Soc 52: 290-296.

Fisher BW, Flowerdew G. 1995. A simple model for predicting postoperative delirium in older patients undergoing elective orthopedic surgery. J Am Geriatr Soc 43: 175-178.

Fries BE, Mehr DR, Schneider D, et al. 1993. Mental dysfunction and resource use in nursing homes. Med Care 31: 898-920.

Hanley J, McNeil BJ. 1982. The meaning and use of the area under an ROC curve. Radiology 143: 129133.

Heagerty PJ, Lumley T, Pepe MS. 2000. Time-dependent ROC curves for censored survival data and a diagnostic marker. Biometrics 56: 337-344.

Ibrahim JG, Chen M-H, Sinha D. 2001. Bayesian survival analysis. New York: Springer.

Inouye SK, Bogardus ST, Charpentier PA, et al. 1999. A multicomponent intervention to prevent delirium in hospitalized older patients. $N$ Engl J Med 340: 669-720.

Inouye SK, S.T B, Baker DI, et al. 2000. The hospital elder life program: a model of care to prevent cognitive and functional decline in older hospitalized patients. J Am Geriatr Soc 48: 16971706.

Inouye SK, VanDyck CH, Alessi CA, et al. 1990. Clarifying confusion: the Confusion Assessment Method, a new method for detection of delirium. Ann Intern Med 113: 941-948. 
Inouye SK, Viscoli CM, Horwitz RI, et al. 1993. A predictive model for delirium in hospitalized elderly medical patients based on admission characteristics. Ann Intern Med 119: 474-481.

Khattree R, Naik DN. 2000. Multivariate data reduction and discrimination with SAS software. Cary, North Carolina: SAS Institut Inc.

Kiely DK, Bergmann MA, Murphy KM, et al. 2003. Delirium among newly admitted postacute facility patients: prevalence, symptoms, and severity. J Gerontol A Biol Sci Med Sci 58: M441-445.

Kiely DK, Jones RN, Bergmann MA, et al. 2007. Association between psychomotor activity delirium subtypes and mortality among newly admitted postacute facility patients. J Gerontol : Med Sci 62A: $174-179$.

Kiely DK, Marcantonio ER, Inouye SK, et al. 2009. Persistent delirium predicts greater mortality. $J$ Am Geriatr Soc 57: 55-61.

Mach J, Kabat V, Olson D, et al. 1996. Delirium and right-hemisphere dysfunction in cognitively impaired older persons. Int Psychogeriatr 8: 373-382.

Madigan D, Raftery AE. 1994. Model selection and accounting for model uncertainty in graphical models using Occam's window. J Am Stat Assoc 89: 1535-1546.

Mahoney FI, Barthel DW. 1965. Functional evaluation: the Barthel Index. MD State Med J 14: 61-65. Marcantonio ER, Goldman L, Mangione CM, et al. 1994. A clinical prediction rule for delirium after elective noncardiac surgery. JAMA 271: 134-139.

Marcantonio ER, Simon SE, Bergmann MA, et al. 2003. Delirium symptoms in post-acute care: Prevalent, persistent, and associated with poor functional recovery. J Am Geriatr Soc 51: 4-9.

McCusker J, Bellavance F, Cardin S, et al. 1999. Detection of older people at increased risk of adverse health outcomes after an emergency visit: the ISAR screening tool. J Am Geriatr Soc 47: 12291237.

McCusker J, Cole MG, Voyer P, et al. 2011a. Prevalence and incidence of delirium in long-term care. Int J Geriatr Psychiatry. DOI:10.1002/gps.2654

McCusker J, Cole MG, Voyer P, et al. 2011b. Use of nurse-observed symptoms of delirium in longterm care: effects on prevalence and outcomes of delirium. Int Psychogeriatr 23: 602-608.

McCusker J, Kakuma R, Abrahamowicz M. 2002. Predictors of functional decline in hospitalized elderly patients: a systematic review. J Gerontol A Biol Sci Med Sci 57: M569-M577.

Ray WA, Taylor JA, Lichtenstein MJ, et al. 1992. The Nursing Home Behavior Problem Scale. J Gerontol 47: M9-M16. 
Rudolph JL, Jones RN, Levkoff SE, et al. 2009. Derivation and validation of a preoperative prediction rule for delirium after cardiac surgery. Circulation 119: 229-236.

Sandberg O, Gustafson Y, Brannstrom B, et al. 1998. Prevalence of dementia, delirium and psychiatric symptoms in various care settings for the elderly. Scand J Soc Med 26: 56-62.

Tombaugh TN, McIntyre NJ. 1992. The Mini-Mental State Examination: a comprehensive review. $J$ Am Geriatr Soc 40: 922-935.

Voyer P, Richard S, Doucet L, et al. 2009. Detecting delirium and subsyndromal delirium using different diagnostic criteria among demented long-term care residents. J Am Med Dir Assoc: 181-188. 